\title{
A!
}

This is an electronic reprint of the original article.

This reprint may differ from the original in pagination and typographic detail.

Petersen, Charlotte F.; Farhan, Alan; Dhuey, Scott; Chen, Zuhuang; Alava, Mikko J.; Scholl, Andreas; Van Dijken, Sebastiaan

Tuning magnetic ordering in a dipolar square-kite tessellation

Published in:

Applied Physics Letters

DOI:

$10.1063 / 1.5014041$

Published: 26/02/2018

Document Version

Publisher's PDF, also known as Version of record

Please cite the original version:

Petersen, C. F., Farhan, A., Dhuey, S., Chen, Z., Alava, M. J., Scholl, A., \& Van Dijken, S. (2018). Tuning magnetic ordering in a dipolar square-kite tessellation. Applied Physics Letters, 112(9), 1-5. [092403]. https://doi.org/10.1063/1.5014041

This material is protected by copyright and other intellectual property rights, and duplication or sale of all or part of any of the repository collections is not permitted, except that material may be duplicated by you for your research use or educational purposes in electronic or print form. You must obtain permission for any other use. Electronic or print copies may not be offered, whether for sale or otherwise to anyone who is not an authorised user. 


\section{Tuning magnetic ordering in a dipolar square-kite tessellation}

Charlotte F. Petersen, Alan Farhan, Scott Dhuey, Zuhuang Chen, Mikko J. Alava, Andreas Scholl, and Sebastiaan van Dijken

Citation: Appl. Phys. Lett. 112, 092403 (2018); doi: 10.1063/1.5014041

View online: https://doi.org/10.1063/1.5014041

View Table of Contents: http://aip.scitation.org/toc/apl/112/9

Published by the American Institute of Physics

\section{Articles you may be interested in}

Control of magnetic vortex polarity by the phase difference between voltage signals

Applied Physics Letters 112, 092404 (2018); 10.1063/1.5020824

Individually grown cobalt nanowires as magnetic force microscopy probes

Applied Physics Letters 112, 092401 (2018); 10.1063/1.4997310

High performance perpendicular magnetic tunnel junction with Co/Ir interfacial anisotropy for embedded and standalone STT-MRAM applications

Applied Physics Letters 112, 092402 (2018); 10.1063/1.5018874

Thin-film topological insulators for continuously tunable terahertz absorption

Applied Physics Letters 112, 091601 (2018); 10.1063/1.5016803

Stressor-layer-induced elastic strain sharing in $\mathrm{SrTiO}_{3}$ complex oxide sheets

Applied Physics Letters 112, 091905 (2018); 10.1063/1.5019920

Investigation of the difference between spin Hall magnetoresistance rectification and spin pumping from the viewpoint of magnetization dynamics

Applied Physics Letters 112, 092406 (2018); 10.1063/1.5017298

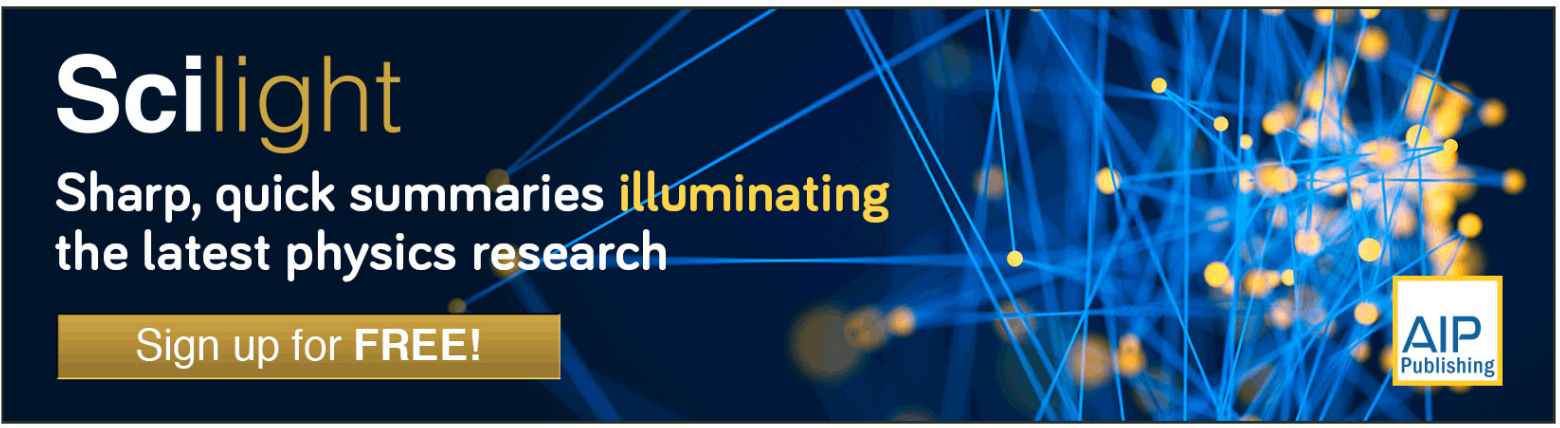




\title{
Tuning magnetic ordering in a dipolar square-kite tessellation
}

\author{
Charlotte F. Petersen, ${ }^{1,2, a)}$ Alan Farhan, ${ }^{3, b)}$ Scott Dhuey, ${ }^{4}$ Zuhuang Chen, ${ }^{5}$ Mikko J. Alava, ${ }^{1}$ \\ Andreas Scholl, ${ }^{3}$ and Sebastiaan van Dijken ${ }^{6}$ \\ ${ }^{1}$ COMP Centre of Excellence, Department of Applied Physics, Aalto University, P.O. Box 11100, \\ FI-00076 Aalto, Espoo, Finland \\ ${ }^{2}$ Institut für Theoretische Physik, Leopold-Franzens-Universität Innsbruck, Technikerstraße 21A, \\ A-6020 Innsbruck, Austria \\ ${ }^{3}$ Advanced Light Source, Lawrence Berkeley National Laboratory (LBNL), 1 Cyclotron Road, Berkeley, \\ California 94720, USA \\ ${ }^{4}$ Molecular Foundry, Lawrence Berkeley National Laboratory (LBNL), 1 Cyclotron Road, Berkeley, \\ California 94720, USA \\ ${ }^{5}$ Department of Materials Science and Engineering, University of California, Berkeley, California 94720, USA \\ ${ }^{6}$ NanoSpin, Department of Applied Physics, Aalto University School of Science, P.O. Box 15100, \\ FI-00076 Aalto, Finland
}

(Received 14 November 2017; accepted 11 February 2018; published online 27 February 2018)

The potential application of artificial spin ice in magnetic nanodevices provides a strong drive to investigate different lattice geometries. Here, we combine components of a recently investigated artificial spin ratchet with components of the prototypical square lattice to form a geometrically frustrated artificial spin ice system where Ising-type nanomagnets are arranged onto a twodimensional square-kite lattice. Using synchrotron-based photoemission electron microscopy, we explore moment configurations achieved in this lattice geometry. Following thermal annealing, we image how a variation of the relevant lattice parameter affects magnetic ordering in four-island squares and four-island vertices during cooling through the Blocking temperature. Depending on lattice spacing, both nearly uniform and disordered spin configurations are accessible in our samples. We show that the relative energies of the building blocks of the system, which are typically used to classify lattice configurations, are not predictive of the low energy states adopted by the experimental system. To understand magnetic ordering in the square-kite lattice, longer range interactions must be considered. Published by AIP Publishing. https://doi.org/10.1063/1.5014041

Frustration generally involves interactions that cannot be simultaneously minimized, leading to extensive ground state degeneracies and the emergence of exotic states. In magnetism, intriguing phenomena reported in systems incorporating frustration ${ }^{1-4}$ have traditionally been explored using macroscopic techniques such as neutron scattering, ${ }^{5}$ Mössbauer spectroscopy, ${ }^{6}$ or heat capacity and susceptibility measurements. ${ }^{2,4}$ The introduction of artificial spin systems has provided a powerful means to investigate frustration microscopically using real-space imaging techniques. ${ }^{7-12}$ One particular interest has focused on the real-time thermodynamics of these systems. ${ }^{13-15}$ In addition, artificial spin ice lattices have shown potential for applications in the fields of spintronics ${ }^{16-18}$ and energy storage. ${ }^{19}$ Most recently, a frustration-by-design ${ }^{20}$ concept involving two-dimensional geometries has been proposed. These lattices exhibit exotic emergent phenomena that do not necessarily occur in nature as a consequence of geometrical or topological frustration. ${ }^{21-28}$ Some of these systems show great potential as functional materials in magnetic nanomotors or actuators. An example is the artificial spin ratchet which can convert energy to unidirectional dynamics in a simple lattice. ${ }^{27}$

Here, we combine the fundamental components of the artificial spin ratchet and the extensively studied square lattice. The artificial spin ratchet consists of square building blocks and shows chiral behavior. We take these squares and

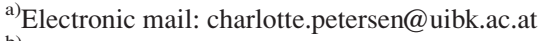

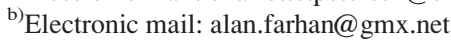

couple them with vertices, with the goal of influencing the ordering within the squares by varying the strength of the coupling. The resulting geometry, a square-kite lattice, is realised through lithographically arranged single-domain Ising-type nanomagnets occupying the centers of the lattice sites (see Fig. 1). The nanomagnets possess a lateral dimension of $450 \times 150 \mathrm{~nm}$ and a thickness of $2.7 \mathrm{~nm}$. As these

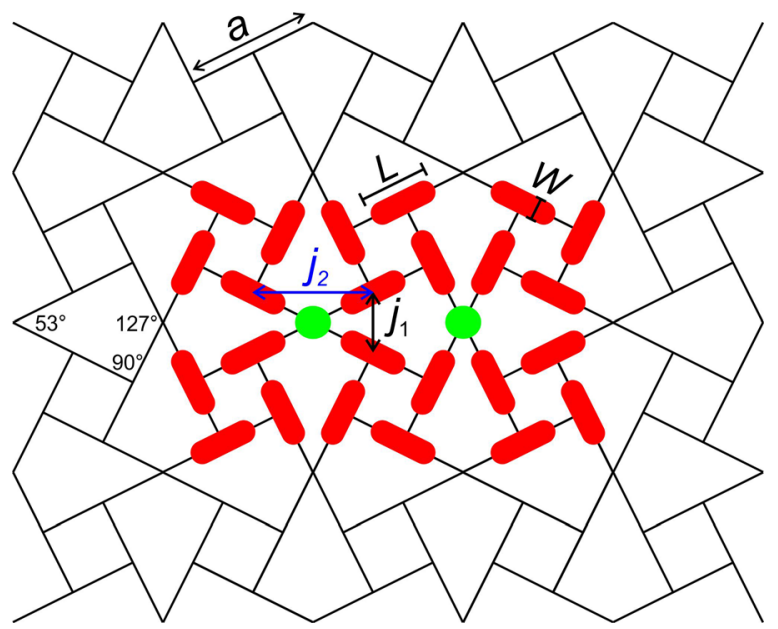

FIG. 1. Schematic of the dipolar square-kite spin ice. The nanomagnets occupy the site centers of a square-kite tessellation. While the nanomagnet dimensions (length $L=450 \mathrm{~nm}$, width $W=150 \mathrm{~nm}$, and thickness $d=2.7 \mathrm{~nm}$ ) are kept constant, the lattice parameter $a$ is varied between $700 \mathrm{~nm}$ and $900 \mathrm{~nm}$. 
nanomagnets are coupled via dipolar magnetic interactions, we refer to this system as the dipolar square-kite spin ice. The thus created lattice consists of two sublattices with horizontal and vertical four-nanomagnet vertices (green circles in Fig. 1). The square components are coupled via fournanomagnet vertices by interactions $J_{1}$ and $J_{2}$. The strength of this coupling is directly tunable by lattice parameter $a$, which we vary from $700 \mathrm{~nm}$ to $900 \mathrm{~nm}$ in this study. We demonstrate that the low-energy configurations and ordering of the system sensitively depend on parameter $a$. Besides, our observations reveal that a whole set of low-energy building blocks is suppressed in favor of moment configurations that minimize second nearest neighbor interactions.

For this work, a $2.7 \mathrm{~nm}$ thick permalloy (Ni81\%Fe19\%) film was patterned using electron beam lithography. Three sets of square-kite lattices were created. Parameter $a$ of the lattices was set to be $700 \mathrm{~nm}, 800 \mathrm{~nm}$, and $900 \mathrm{~nm}$, respectively. A $2 \mathrm{~nm}$ thick Al capping layer was added to avoid fast oxidation. Magnetic configurations were directly visualized using synchrotron-based photoemission electron microscopy (PEEM), ${ }^{29}$ employing $\mathrm{X}$-ray magnetic circular dichroism (XMCD) at the Fe $L_{3}$ edge. ${ }^{30}$ Similar to previous work, ${ }^{13,23,24}$ a film thickness of $2.7 \mathrm{~nm}$ was chosen to ensure that thermally driven moment fluctuations can be resolved with XMCD imaging (7-9s per image) at experimentally accessible temperatures. In our particular case, the blocking temperature $T_{B}$ of the nanomagnets was $280 \mathrm{~K}$.

In analogy to other artificial spin ice systems,, ,22-24,31 moment configurations achieved in the dipolar square-kite lattice are characterized by its building blocks, four-island squares, and four-island vertices in this geometry. Possible square- and vertex types are listed with increasing dipolar energy in Fig. 2 from A to D and I to IV, respectively. In our model, we assume each nanomagnet to have uniform magnetic moment density $|\mathbf{m}| / L$ with its width being negligible relative to its length. Thus, each nanomagnet is replaced by an infinitesimally thin compass needle with a length $L=450 \mathrm{~nm}$. This is equivalent to placing a pair of magnetic charges $\pm q$ at the tips of the nanomagnet. ${ }^{32,33}$ The interaction Hamiltonian is then given by

$$
\begin{aligned}
H_{i, j}= & \frac{\mu_{0}|\mathbf{m}|^{2}}{4 \pi L^{2}}\left[\frac{1}{\left|\mathbf{r}_{a_{i}}-\mathbf{r}_{a_{j}}\right|}-\frac{1}{\left|\mathbf{r}_{a_{i}}-\mathbf{r}_{b_{j}}\right|}\right. \\
& \left.-\frac{1}{\left|\mathbf{r}_{b_{i}}-\mathbf{r}_{a_{j}}\right|}+\frac{1}{\left|\mathbf{r}_{b_{i}}-\mathbf{r}_{b_{j}}\right|}\right]
\end{aligned}
$$

where $\mathbf{r}_{a_{i}}$ and $\mathbf{r}_{b_{i}}$ are the locations of the positive and negative charges on nanomagnet $i$, respectively. $\mu_{0}=4 \pi \times 10^{-7} \mathrm{NA}^{-2}$ is the magnetic permeability, and $|\mathbf{m}|=M=M V / V$ is the magnetic moment of a nanomagnet, with $M$ being the saturation magnetization of the patterned magnetic film and $V$ the volume of the nanomagnet. The energies in Fig. 2 are calculated for a lattice with $a=700 \mathrm{~nm}$ and $M=486 \mathrm{kA} / \mathrm{m}$.

Based on these calculations, we see that Type A and Type B squares are almost identical in energy, while Type I vertices have significantly lower energy compared to other possible vertex configurations, similar to artificial square ice. ${ }^{14}$ In contrast to artificial square ice, where the so-called Type II vertices are four-fold degenerate, ${ }^{8}$ the Type II vertex

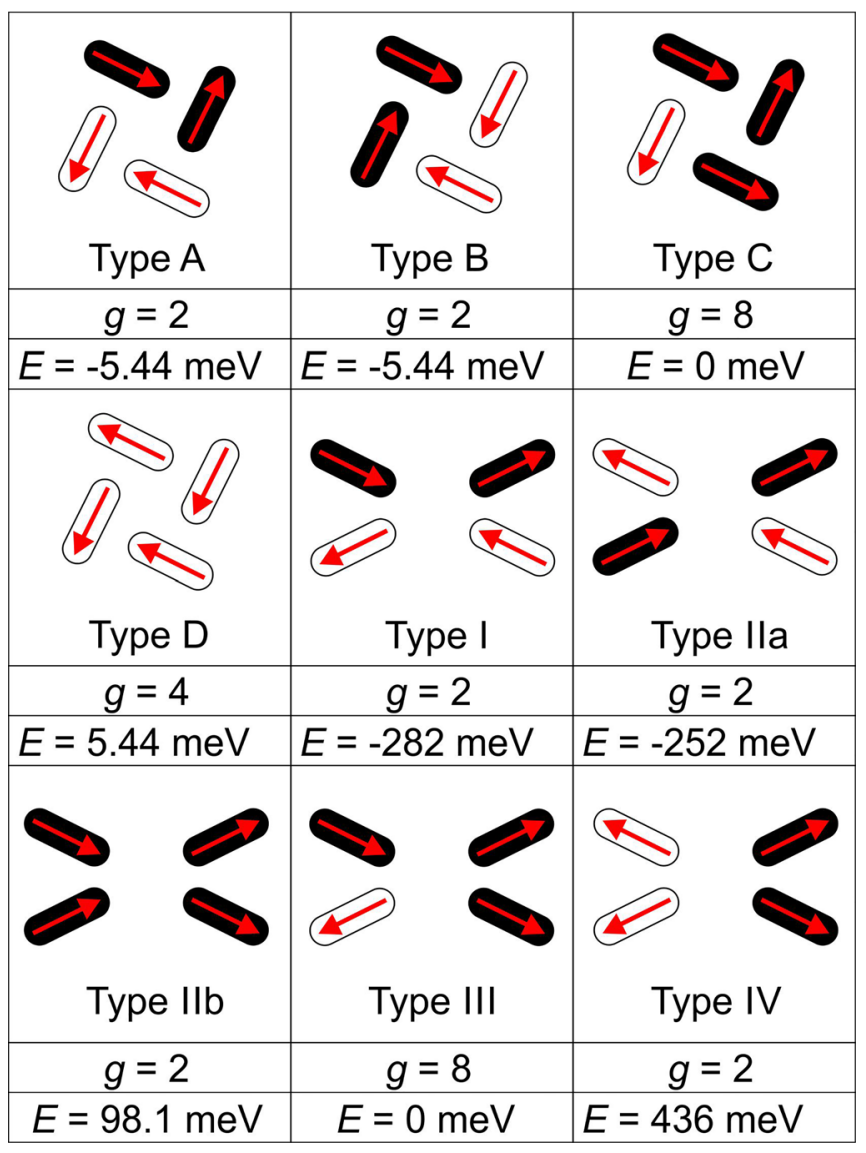

FIG. 2. Square- and vertex types of the dipolar square-kite spin ice. They are shown with their corresponding dipolar energies $E$ and degeneracies $g$.

configurations in the dipolar square-kite lattice are split into two different types, with Type IIa being lower in energy than Type IIb. This can be easily understood by considering that Type IIa configurations minimize the stronger $J_{1}$ vertex interactions, while Type IIb states satisfy the weaker $J_{2}$ vertex interactions (see Fig. 1). The Type III and Type IV states exhibit the same degeneracies as in artificial square ice.

To experimentally measure low-energy configurations, we placed our spin ice lattices $(a=700,800$, and $900 \mathrm{~nm})$ into the PEEM. There, the arrays were first heated to $330 \mathrm{~K}$, and after a waiting period of $2-3 \mathrm{~h}$, the sample was cooled down below the blocking point to $250 \mathrm{~K}$, so that low-energy configurations could be imaged [see Figs. 3(a), 3(b), and 3(c)]. The obtained magnetic configurations are characterized by plotting the square- and vertex type populations as a function of lattice parameter $a$ [see Figs. 3(d) and 3(e)]. To obtain good statistics, we repeated this annealing protocol 5 times. As expected, Type I vertices dominate the configurational landscape. As $a$ changes from $700 \mathrm{~nm}$ to $900 \mathrm{~nm}$, the population of Type I shows only a moderate decrease, while the Type IIa vertex population rises, correspondingly. All other vertex types (Type IIb, III, and IV) are almost never observed, as they are energetically unfavorable. The square type populations show a more pronounced variation, as the Type A population rapidly drops with the increasing lattice parameter, while the populations of Type $\mathrm{C}$ and Type D squares rise accordingly until a highly disordered moment configuration is achieved at $a=900 \mathrm{~nm}$. In this disordered state, the populations of Type A, C, and D squares are almost 

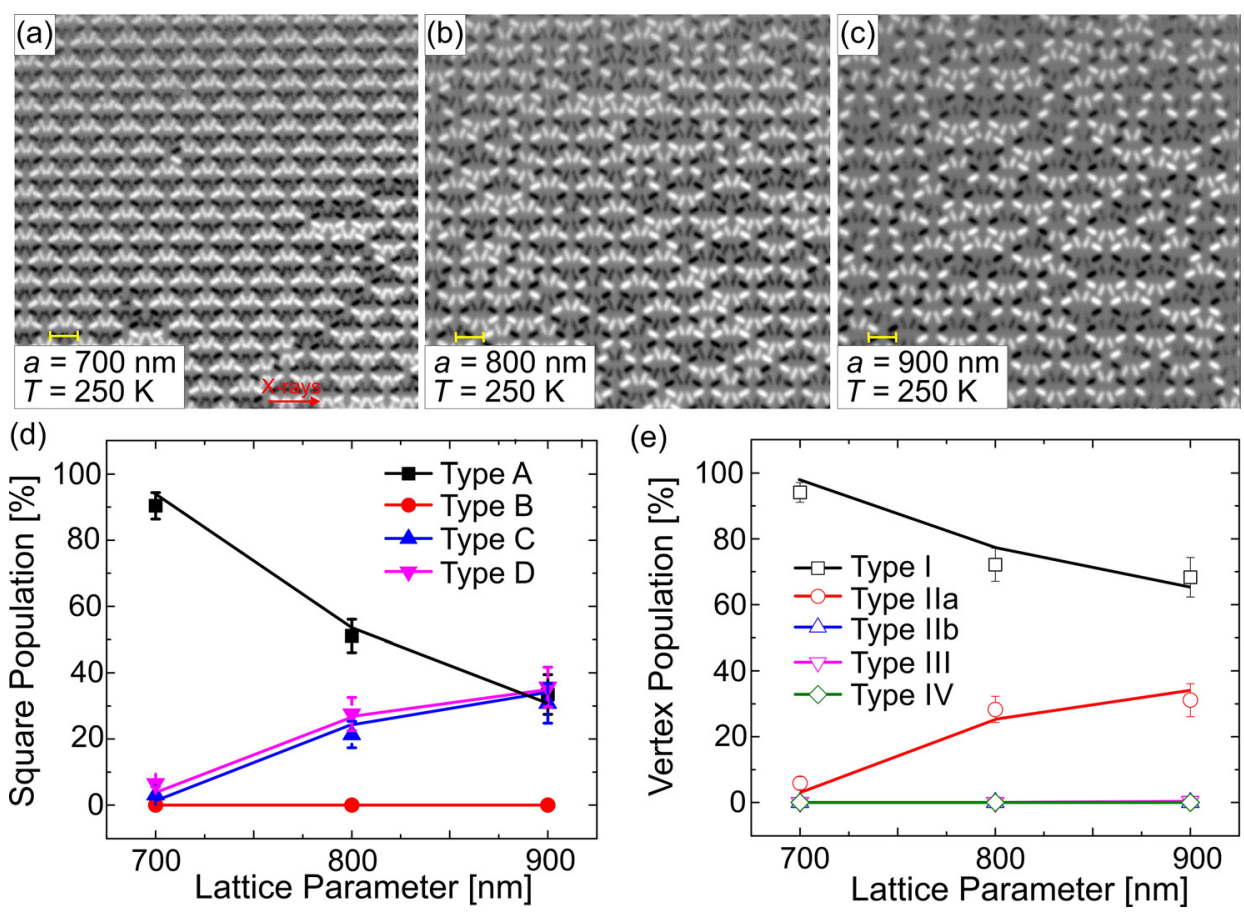

FIG. 3. (a), (b), and (c) XMCD images of frozen-in low-energy configurations of the dipolar square-kite spin ice with lattice spacings $a=700 \mathrm{~nm}, 800 \mathrm{~nm}$, and $900 \mathrm{~nm}$, respectively. The images are obtained following a thermal annealing procedure at which the sample was kept at a constant temperature of $330 \mathrm{~K}$ (blocking point $T_{B}=280 \mathrm{~K}$ ) for $2-3 \mathrm{~h}$, before it was cooled down to $250 \mathrm{~K}$ for XMCD imaging. The obtained XMCD contrast is a direct measure of the projection of the magnetization on the X-ray polarization vector. Moments pointing towards the incoming $\mathrm{x}$-rays will appear dark, while moments pointing against the incoming $\mathrm{X}$-rays will appear bright. The yellow bar corresponds to a length of $1 \mu \mathrm{m}$. (d) Average square type population plotted as a function of lattice parameter $a$. (e) Average vertex type population plotted as a function of $a$. The error bars in (d) and (e) correspond to the standard deviation, resulting from five annealing procedures on the same arrays. The simulation results are included as lines. equal. The Type D square is generated in the artificial spin ratchet through application of a magnetic field. Here, we observe it with no applied field, at large values of $a$.

Interestingly, Type B squares are never observed for all lattice spacings, despite having the same energy as Type A squares (see Fig. 2). At first sight, this observation seems surprising, as both Type A/Type I and Type B/Type I configurations are possible and minimize the relevant vertex interactions ( $J_{1}$ and $J_{2}$ in Fig. 1 ). We investigated this curiosity by including a varying number of interactions in computer simulations of the lattice. We perform Monte Carlo simulations using the Hamiltonian given in Eq. (1). The saturation magnetization was selected to fit the experimental results. We used a temperature of $T=250 \mathrm{~K}$, as in the experiments. The simulated lattice consisted of 14400 nanomagnets. The results from simulations taking into account all interactions are included as lines in the plots of Fig. 3. In this case, the experiments and simulations are consistent. However, if only nearest neighbor interactions in a nanomagnet's own vertex or square are considered, we find different populations compared to the experiments. In this case, a significant population of Type B squares is present in the simulations [see Fig. 4(b)]. Only if second nearest neighbor interactions are included in the simulations, do we qualitatively reproduce the experimental populations, with almost no Type B squares [see Fig. 4(d)]. This implies that second nearest neighbor interactions favor the formation of Type $A$ squares and, consequently, that the energies of the vertex and square building blocks are not sufficient to predict low energy configurations of the dipolar square-kite lattice.

To further probe the long-range behavior of the lattice, we calculated the spin correlations between islands (Fig. 5). The presented data are based on simulation. Experimental values (not shown) are in good agreement with the simulations [see also Figs. 3(d) and 3(e)] but have less statistics. Spins in the same direction $\left(\mathbf{S}_{1} \cdot \mathbf{S}_{2}>0\right.$, where $\mathbf{S}_{1}$ and $\mathbf{S}_{2}$ are the spin vectors) are assigned a correlation value of 1 , while spins in opposite directions are assigned $-1 .^{9}$ If the spins are perpendicular, the pair is assigned a correlation of 1 if $\left(\mathbf{S}_{1} \cdot \mathbf{v}_{12}\right) \times\left(\mathbf{S}_{2} \cdot \mathbf{v}_{12}\right)>0$, where $\mathbf{v}_{12}$ is the vector between spins 1 and 2 . If this expression is 0 , the correlation is not calculated and is plotted as 0 . The color of each island in Fig. 5 is given by its correlation with the central island

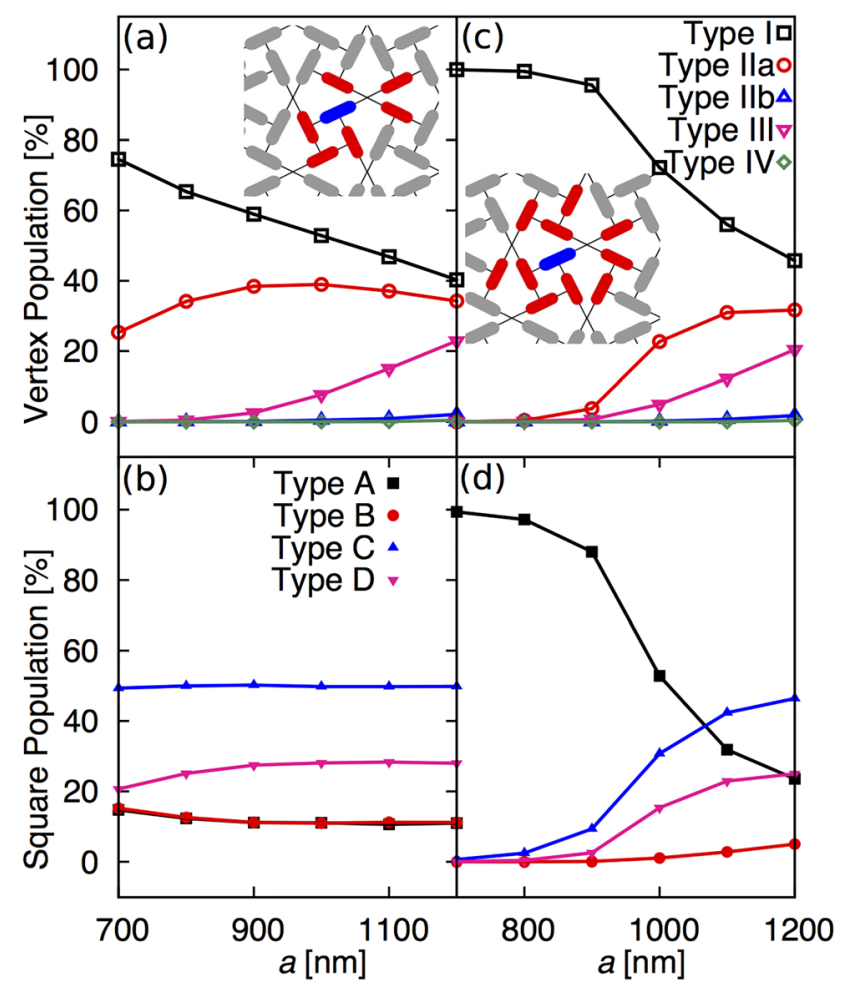

FIG. 4. Simulated vertex and square populations using small interaction cutoffs. In (a) and (b), vertex and square populations, respectively, are plotted from a simulation including only nearest neighbor interactions. In (c) and (d), vertex and square populations, respectively, are plotted when the simulations additionally include second nearest neighbors. The interactions included in each system are indicated in the respective insets. The blue island interacts with only the islands colored in red. 

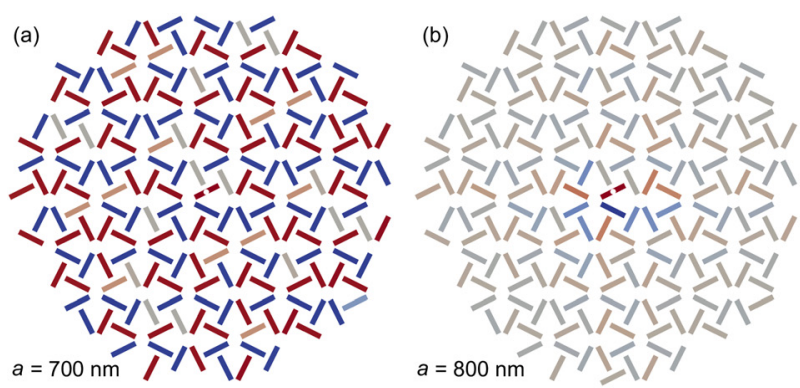

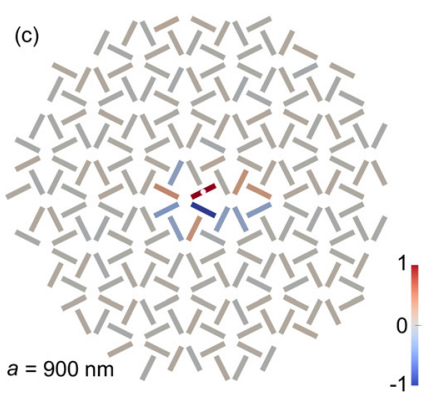

FIG. 5. Correlation between pairs of spins. The colour of each island indicates its average correlation with the central island (marked by a white circle), calculated from the simulation configurations. (a), (b), and (c) correspond to systems with lattice parameters $700 \mathrm{~nm}$, $800 \mathrm{~nm}$, and $900 \mathrm{~nm}$ respectively. (marked with a white circle), averaged over all equivalent pairs in the lattice. We clearly observe strong long-range ordering in the $a=700 \mathrm{~nm}$ system. At $a=900 \mathrm{~nm}$, the ordering of squares is drastically reduced, while nearest neighbor islands within a vertex remain strongly correlated by the ice rule.

In summary, we introduced an artificial frustrated system whose low energy moment configurations can be tuned by a change in lattice parameter $a$. With decreasing coupling (increasing $a$ ), we see a transition from long-range to shortrange order. We find that it is not possible to predict the low energy configurations of the system by considering only the building blocks of the lattice. A good agreement between experiments and simulations is only attained when longer range interactions are taken into account, highlighting their importance in the magnetic ordering of this and similar types of artificial spin ice systems. While the dipolar squarekite lattice offers an interesting artificial frustrated system from a fundamental aspect, as it combines features from the artificial spin ratchet $^{27}$ and artificial square ice 8,14 with an interesting capability of tuning the ground state preferences, it also offers an interesting prospect for researchers interested in exploring its dynamic response. ${ }^{16-18,34}$ So far, such studies focused on vertex-dominated artificial spin ice geometries, ${ }^{16-18}$ and it will be interesting to see how tunable square-type preferences in the square-kite lattice influence the dynamic properties of respective systems. Artificial spin ices can also be used as programmable metamaterials for spin waves, where the magnon band structure is sensitive to the moment configuration. ${ }^{17}$ The ability to tune the degree of long-range ordering in the square-kite lattice makes it a promising candidate for future explorations in magnonics and spintronics.

The authors would like to thank Michael Elowson for his support. This work was supported by the Swiss National Science Foundation, and part of this work was performed at Advanced Light Source (ALS) and the Molecular Foundry, Lawrence Berkeley National Laboratory (LBNL), 1 Cyclotron Road, Berkeley, CA 94720, USA. The ALS and the Molecular Foundry are supported by the Director, Office of Science, Office of Basic Energy Sciences, of the U.S. Department of Energy under Contract No. DE-AC0205CH11231. C.F.P. and M.J.A. are supported by the Academy of Finland through its Centres of Excellence Programme (2012-2017) under Project No. 251748 and the FiDiPro program, Project 13282993. They acknowledge the computational resources provided by the Aalto Science-IT project.

${ }^{1}$ M. J. Harris, S. T. Bramwell, D. F. McMorrow, T. Zeiske, and K. W. Godfrey, Phys. Rev. Lett. 79, 2554 (1997).

${ }^{2}$ A. P. Ramirez, A. Hayashi, R. J. Cava, R. Siddharthan, and B. S. Shastry, Nature 399, 333 (1999).

${ }^{3}$ C. Castelnovo, R. Moessner, and S. L. Sondhi, Nature 451, 42 (2008).

${ }^{4}$ J. Snyder, J. S. Slusky, R. J. Cava, and P. Schiffer, Nature 413, 48 (2001).

${ }^{5}$ D. J. Morris, D. A. Tennant, S. A. Grigera, B. Klemke, C. Castelnovo, and R. Moessner, Science 326, 411 (2009).

${ }^{6}$ J. van Lierop and D. H. Ryan, Phys. Rev. Lett. 86, 4390 (2001).

${ }^{7}$ Y. Han, Y. Shokef, A. M. Alsayed, P. Yunker, T. C. Lubensky, and A. G. Yodh, Nature 456, 898 (2008).

${ }^{8}$ R. F. Wang, C. Nisoli, R. S. Freitas, J. Li, W. McConville, B. J. Cooley, M. S. Lund, N. Samarth, C. Leighton, V. H. Crespi, and P. Schiffer, Nature 439, 303 (2006).

${ }^{9}$ Y. Qi, T. Brintlinger, and J. Cumings, Phys. Rev. B 77, 094418 (2008).

${ }^{10}$ S. Ladak, D. E. Read, G. K. Perkins, L. F. Cohen, and W. R. Branford, Nat. Phys. 6, 359 (2010).

${ }^{11}$ J. P. Morgan, A. Stein, S. Langridge, and C. H. Marrows, Nat. Phys. 7, 75 (2011).

${ }^{12}$ I. A. Chioar, B. Canals, D. Lacour, M. Hehn, B. S. Burgos, T. O. Menteş, A. Locatelli, F. Montaigne, and N. Rougemaille, Phys. Rev. B 90, 220407(R) (2014).

${ }^{13}$ A. Farhan, P. M. Derlet, A. Kleibert, A. Balan, R. V. Chopdekar, M. Wyss, L. Anghinolfi, F. Nolting, and L. J. Heyderman, Nat. Phys. 9, 375 (2013).

${ }^{14}$ A. Farhan, P. M. Derlet, A. Kleibert, A. Balan, R. V. Chopdekar, M. Wyss, J. Perron, A. Scholl, F. Nolting, and L. J. Heyderman, Phys. Rev. Lett. 111, 057204 (2013).

${ }^{15}$ V. Kapaklis, U. B. Arnalds, A. Farhan, R. V. Chopdekar, A. Balan, A. Scholl, L. J. Heyderman, and B. Hjörvarsson, Nat. Nanotechnol. 9, 514 (2014).

${ }^{16}$ S. Gliga, A. Kákay, R. Hertel, and O. G. Heinonen, Phys. Rev. Lett. 110, 117205 (2013).

${ }^{17}$ E. Iacocca, S. Gliga, R. L. Stamps, and O. Heinonen, Phys. Rev. B 93, 134420 (2016).

${ }^{18}$ M. B. Jungfleisch, W. Zhang, E. Iacocca, J. Sklenar, J. Ding, W. Jiang, S. Zhang, J. E. Pearson, V. Novosad, J. B. Ketterson, O. Heinonen, and A. Hoffmann, Phys. Rev. B 93, 100401(R) (2016).

${ }^{19}$ E. Y. Vedmedenko, Phys. Rev. Lett. 116, 077202 (2016).

${ }^{20}$ I. Gilbert, C. Nisoli, and P. Schiffer, Phys. Today 69(7), 54 (2016).

${ }^{21}$ V. S. Bhat, J. Sklenar, B. Farmer, J. Woods, J. T. Hastings, S. J. Lee, J. B. Ketterson, and L. E. De Long, Phys. Rev. Lett. 111, 077201 (2013).

${ }^{22}$ I. Gilbert, G.-W. Chern, S. Zhang, L. O’Brien, B. Fore, C. Nisoli, and P. Schiffer, Nat. Phys. 10, 670 (2014).

${ }^{23}$ I. Gilbert, Y. Lao, I. Carrasquillo, L. O'Brien, J. D. Watts, M. Manno, C. Leighton, A. Scholl, C. Nisoli, and P. Schiffer, Nat. Phys. 12, 162 (2016).

${ }^{24}$ A. Farhan, A. Scholl, C. F. Petersen, L. Anghinolfi, C. Wuth, S. Dhuey, R. V. Chopdekar, P. Mellado, M. J. Alava, and S. van Dijken, Nat. Commun. 7, 12635 (2016).

${ }^{25}$ J. Drisko, T. Marsh, and J. Cumings, Nat. Commun. 8, 14009 (2017).

${ }^{26}$ B. Farmer, V. S. Bhat, A. Balk, E. Teipel, N. Smith, J. Unguris, D. J. Keavney, J. T. Hastings, and L. E. De Long, Phys. Rev. B 93, 134428 (2016).

${ }^{27}$ S. Gliga, G. Hrkac, C. Donnelly, J. Büchi, A. Kleibert, J. Cui, A. Farhan, E. Kirk, R. V. Chopdekar, Y. Masaki, N. S. Bingham, A. Scholl, R. L. Stamps, and L. J. Heyderman, Nat. Mater. 16, 1106 (2017). 
${ }^{28}$ A. Farhan, C. F. Petersen, S. Dhuey, L. Anghinolfi, Q.-H. Qin, M. Saccone, S. Velten, C. Wuth, S. Gliga, P. Mellado, M. J. Alava, A. Scholl, and S. van Dijken, Nat. Commun. 8, 995 (2017).

${ }^{29}$ A. Doran, M. Church, T. Miller, G. Morrison, A. T. Young, and A. Scholl, J. Electron Spectrosc. Related Phenom. 185, 340 (2012).

${ }^{30}$ J. Stöhr, Y. Wu, B. D. Hermsmeier, M. G. Samant, G. R. Harp, S. Koranda, D. Dunham, and B. P. Tonner, Science 259, 658 (1993).
${ }^{31}$ L. A. S. Mól, A. R. Pereira, and W. A. Moura-Melo, Phys. Rev. B 85, 184410 (2012).

${ }^{32}$ G. Möller and R. Moessner, Phys. Rev. B 80, 140409(R) (2009).

${ }^{33}$ G. W. Chern, P. Mellado, and O. Tchernyshyov, Phys. Rev. Lett. 106, 207202 (2011).

${ }^{34}$ J. Park, B. L. Le, J. Sklenar, G. W. Chern, J. D. Watts, and P. Schiffer, Phys. Rev. B 96, 024436 (2017). 